Short Communications

\title{
Polymerase Chain Reaction: A Better Method for Diagnosing Chronic Schistosoma mansoni Infections
}

\author{
Ekhlas Hamed Abdel-Hafeez ${ }^{1}$, Rabie M. Mohamed ${ }^{1}$, Usama S. Belal ${ }^{1}$, Ehab M. Abdel-Raheem², Koji Naoi ${ }^{3}$ \\ and Kazumi Norose ${ }^{4 *}$ \\ Received 15 May, 2015 Accepted 11 July, 2015 Published online 25 July, 2015
}

\begin{abstract}
For more effective diagnosis of the acute and chronic stages of Schistosoma mansoni infection in humans, the polymerase chain reaction (PCR) technique was compared with the Kato-Katz method. A total of 150 stool samples were collected from inpatient and outpatient clinics at the Department of Tropical Medicine, Minia University Hospital, Egypt. Three groups of patients, 50 with acute intestinal schistosomiasis, 70 with chronic intestinal schistosomiasis and 30 normal healthy controls were studied. Stool samples were analyzed by PCR and the Kato-Katz method. The mean number of eggs per gram of feces was 4.6 when estimated by the Kato-Katz method in positive stool samples from acute schistosomiasis cases but only 1.7 in chronic cases. In acute intestinal schistosomiasis, 15 and 45 out of 50 cases were positive by Kato-Katz and PCR, respectively. In the chronic intestinal schistosomiasis cases, 6 and 68 out of 70 cases were positive by the Kato-Katz and PCR methods, respectively. We conclude that PCR appears to be an effective diagnostic technique for $S$. mansoni infection, especially where a low worm burden exists, such as in chronic cases.
\end{abstract}

Key words: S. mansoni, Kato-Katz method, PCR

\section{INTRODUCTION}

The worldwide burden of schistosomiasis has been expressed as the number of infected individuals (200-209 million) and the number of inhabitants at risk of infection residing in schistosomiasis-endemic countries $(600-779$ million) [1-4]. The Global Burden of Disease Study currently attributes 14,000 deaths to schistosomiasis every year [5]. Annually, the overall number of disabilityadjusted life-years lost due to schistosomiasis is around 1.532 million, $77 \%$ of which are in sub-Saharan Africa [5].

The infection intensity of schistosomiasis and the morbidities associated with it have diminished in the wake of chemotherapy- and community-based control programs $[6,7]$. Parasite transmission occurs in areas of high endemicity, while reinfection and ongoing morbidities such as anemia and malnutrition are not uncommon. This emphasizes the need for more efficient methods of diagnosis, as the methods in current use are less sensitive at estimating the prevalence of infection and parasite burden $[8,9]$.

Finding eggs in the stool of an infected patient is the final diagnosis for Schistosoma mansoni infection [10]. As the Kato-Katz method [11] is relatively inexpensive and simple, it is currently the technique of choice for definitive diagnosis of $S$. mansoni. However, this parasitological method is not sensitive enough for use in areas of low transmission with low infection intensities. Moreover, parasitological techniques are inadequate for both the diagnosis of recent infections when the worms have not yet produced eggs [10] and for the late stage of the disease when limited egg numbers are passed in stools. With the development of molecular biological techniques, this problem can be overcome by identifying parasite antigens circulating in an infected person [12].

Among the molecular biological techniques that target nucleic acids, polymerase chain reaction (PCR) has emerged as one of the most common diagnostic tools in human medicine [13]. In terms of advancing parasite de-

\footnotetext{
${ }^{1}$ Department of Parasitology, Faculty of Medicine, Minia University, Minia 61519, Egypt

${ }^{2}$ Department of Tropical Medicine, Minia University Hospital, Minia University, Minia 61519, Egypt

${ }^{3}$ Medical Corporation Kohitsujikai, Chiba 275-0001, Japan

${ }^{4}$ Department of Infection and Host Defense, Graduate School of Medicine, Chiba University, Chiba 260-8670, Japan

*Corresponding author:

Department of Infection and Host Defense, Graduate School of Medicine, Chiba University, Chiba 260-8670, Japan

Tel: +81-43-226-2073

Fax: +81-43-226-2076

E-mail: norose@faculty.chiba-u.jp
} 
tection and disease diagnosis, PCR has played a substantial role [14]. Hamburger et al. developed a PCR procedure for amplification of a highly repetitive DNA sequence in the parasite when examining $S$. mansoni infestation in water $[15,16]$. Pontes et al. detected $S$. mansoni DNA in human serum and feces for the first time by PCR using the same primers described by Hamburger et al. [10].

Here, we assessed the effectiveness of PCR as a technique for the diagnosis of $S$. mansoni infection, especially in the chronic stage of infection, and compared it to the Kato-Katz method [11]. The sensitivity and specificity of each method were investigated.

\section{Methods}

\section{Patients}

In total, 150 fecal samples were collected from inpatient and outpatient clinics at the Department of Tropical Medicine, Minia University Hospital, Egypt, after the patients verbally agreed to enrolment in this study. The study was reported to the Research Ethics Committee of the Faculty of Medicine, Minia University. In terms of its constitution, this committee operates according to the International Conference on Harmonization of Good Clinical Practice guidelines. Group 1 comprised 50 patients (mean $\pm \mathrm{SD}=13.60 \pm 2.15$ years of age, all males) diagnosed clinically as having acute intestinal schistosomiasis based on complaints of diarrhea (generally associated with abdominal pain), tenesmus, and the passage of bloody stools (bilharzial dysentery) 1-2 months after swimming and following exclusion of diagnoses different from schistosomiasis. Diarrhea, dysentery, and blood in stool were scored as follows: 0 , no; 1 , mild; 2 , moderate; 3 , severe; the mean scores for these symptoms were $1.78 \pm 0.86,1.80 \pm 0.90$, and $1.60 \pm 0.93$, respectively. Physical condition was scored as follows: 1, healthy; 2, good condition; 3, pale; 4, weak and pale and the mean score was $1.90 \pm 0.89$. History of swimming in a canal over a period of one week was scored as follows: 0 , no; 1 , once; 2 , twice; 3 , three times or more, and the mean score was $1.62 \pm 0.70$. The duration of the complaints was also recorded and the mean value was $16.24 \pm 10.11$ days.

Group 2 comprised 70 patients $(55.19 \pm 7.69$ years of age, 53 males and 17 females) who were diagnosed clinically as having chronic intestinal schistosomiasis (past history of bilharziasis with or without anti-bilharzial treatment) based on positive anti-S. mansoni antibodies and presence of clinical symptoms typical of this disease (i.e., hepatomegaly, splenomegaly, esophageal varices, and ascites). Hepatomegaly was scored as follows: 0, normal or no extension below the costal margin; 1, mild (extended liver, as measured in centimeters or finger breadth of 2 or 5 $\mathrm{cm}$ ); 2, moderate (extended liver; score between 1 and 3); 3 , huge hepatomegaly reaching to the right iliac fossa. Splenomegaly was scored as follows: 0 , none; 1 , spleen is palpable under the costal margin; 2, spleen is palpable between the costal margin and umbilicus and 3; spleen is palpable below the umbilicus. Esophageal varices were scored as follows: 0 , none; 1 , dilated veins $(<5 \mathrm{~mm})$ still at the level of the surrounding tissue; 2, dilated, straight veins $(>5 \mathrm{~mm})$ protruding into the esophageal lumen but not obstructing it; 3, large, tense and winding veins already obstructing the esophageal lumen considerably; 4, near complete obstruction of the esophageal lumen with impending danger of hemorrhage (cherry-red spots). Ascites was scored as follows [17]: 0, none; 1 , mild, only visible on ultrasound and CT; 2, detectable with flank bulging and shifting dullness; 3 , directly visible, confirmed with the fluid wave/thrill test. The mean physical condition score, determined the same way as in the first group, was $3.61 \pm$ 0.49 . The mean duration of complaints was $86.24 \pm 42.45$ days. The average scores for hepatomegaly, splenomegaly, esophageal varices, and ascites were as follows: $0.21 \pm$ $0.59,1.26 \pm 0.53,0.19 \pm 0.55$ and $0.24 \pm 0.67$, respectively.

Group 3 consisted of 30 normal healthy controls (10 20 years of age, all males) who were selected from the same schistosomiasis endemic area. Past and present histories, clinical examinations, and ultrasonography confirmed them to be healthy.

\section{Methods}

A clean, plastic container was given to each individual and one fecal sample per subject was collected. Fecal specimens were kept on ice and transported to the Parasitology Laboratory, College of Medicine, Minia University. After Kato-Katz stool examinations [11], the remaining samples were stored at $-70^{\circ} \mathrm{C}$ until further use. Stool samples were collected on 3 successive days for all groups and were examined by PCR (one test per sample, see below). The results represent the mean $\pm \mathrm{SD}$. Differences between the acute and chronic cases were analyzed by Student's $t$ test for the mean number of eggs per gram (EPG) and by Fisher's exact probability test for the statistical significance of the PCR assay. $P<0.05$ was regarded as significant.

Stool samples were assessed for the presence of $S$. mansoni eggs by the Kato-Katz method according to Katz et al. [11]. Three glass slides (43 $\mathrm{mg}$ of feces per slide) were arranged for assessing each sample, and the average number of EPG of stool was calculated.

Preparation of stool samples prior to extraction of 
DNA involved mixing $\sim 0.5 \mathrm{~g}$ of stool with $1.0 \mathrm{~mL}$ of distilled water in a $1.5-\mathrm{mL}$ microcentrifuge tube with vigorous agitation, followed by centrifugation at $500 \times \mathrm{g}$ for 2 min. After supernatant removal, the pellet was washed again as before, except that the sample was also gently agitated for $10 \mathrm{~min}$ to rupture any $S$. mansoni eggs present before centrifugation [18]. To isolate parasite DNA, the pellet was subjected to the following modification of the rapid one-step extraction method [19]. Briefly, $0.7 \mathrm{~mL}$ of ROSE buffer (10 mM Tris, pH 8.0, 300 mM EDTA, pH $8.0,1 \%$ sodium lauryl sulfate, $1 \%$ polyvinylpolypyrrolidone) was added to the washed fecal pellet and the tube was heated to $95^{\circ} \mathrm{C}$ for $20 \mathrm{~min}$. After the first $10 \mathrm{~min}$ of heating, the sample was agitated forcefully, centrifuged at $14,000 \times g$ for $10 \mathrm{~min}$ at room temperature, and the supernatant moved to a clean microcentrifuge tube. In a separate tube, $500 \mu \mathrm{L}$ of this supernatant was mixed with $50 \mu \mathrm{L}$ of cold $\left(4^{\circ} \mathrm{C}\right) 3 \mathrm{M}$ sodium acetate, $\mathrm{pH} 5.3$, and $1 \mathrm{~mL}$ of cold $\left(-20^{\circ} \mathrm{C}\right)$ ethanol. The mixture was centrifuged immediately at $14,000 \times g$ for $15 \mathrm{~min}$. After discarding the supernatant, the pellet was washed with $500 \mu \mathrm{L}$ of $70 \%$ ethanol $\left(-20^{\circ} \mathrm{C}\right)$ once, followed by centrifugation at $14,000 \times \mathrm{g}$ for $10 \mathrm{~min}$. After discarding the supernatant, the pellet was dried at $37^{\circ} \mathrm{C}$ for $15 \mathrm{~min}$. Cold $\left(4^{\circ} \mathrm{C}\right)$ buffer $(100 \mu \mathrm{L} ; 10$ $\mathrm{mM}$ Tris-HCl, $\mathrm{pH} 8.0,1 \mathrm{mM}$ EDTA, $\mathrm{pH}$ 8.0) was used to re-suspend the pellet.

For the PCR assay, the primers were designed to amplify the 121-basepair tandem repeat sequence of S. mansoni [16]. DNA extracted from the fecal samples was diluted $1: 100$, and $1 \mu \mathrm{L}$ was used as the template in a 10 $\mu \mathrm{L}$ final volume containing $20 \mathrm{mM}$ Tris- $\mathrm{HCl}, \mathrm{pH} 8.4,50$ $\mathrm{mM} \mathrm{KCl}, 1.5 \mathrm{mM} \mathrm{MgCl} 2,0.5 \mu \mathrm{M}$ of each primer, $200 \mu \mathrm{M}$ of the four dNTPs, and 0.75 units of Taq DNA polymerase (Promega Corp., Egypt). In total, 35 amplification cycles were used, following the method of Pontes et al. [10]. Positive ( $S$. mansoni egg DNA) and negative controls were included in the assay. The PCR products were electrophoresed on $2 \%$ agarose gels and stained with ethidium bromide.

\section{RESULTS}

The mean EPG value calculated by the Kato-Katz method [11] in S. mansoni-positive stool samples for the acute and chronic cases was $4.60 \pm 1.39$ and $1.70 \pm 0.42$, respectively (Fig. 1A). The mean EPG value was significantly $(P<0.01)$ lower in the chronic cases than in the acute cases. The number of positive cases observed using the Kato-Katz method was 15 of 50 cases $(30 \%)$ in the acute intestinal schistosomiasis group and 6 of 70 cases $(8.6 \%)$ in the chronic schistosomiasis group (Table 1). Group 3 had no Schistosoma eggs or eggs related to other parasitic infections.

PCR-amplified 121-bp bands were observed in the parasite-positive samples, but the parasite-negative samples showed no bands (Fig. 1B). The number of positive cases observed using the PCR technique was higher than that detected by the Kato-Katz stool examination method, with 45 of 50 cases $(90 \%)$ detected in the acute intestinal

A

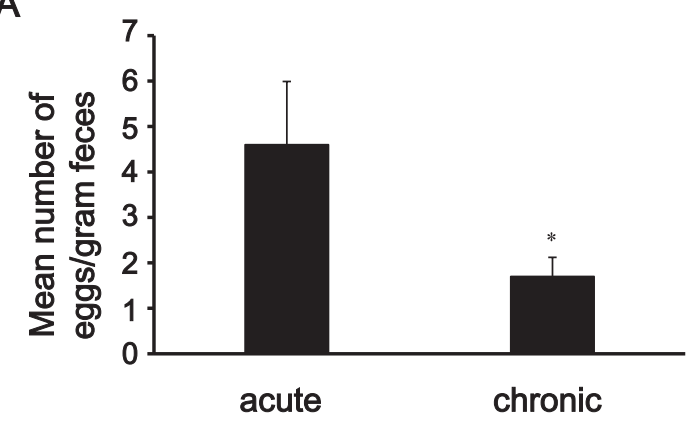

B

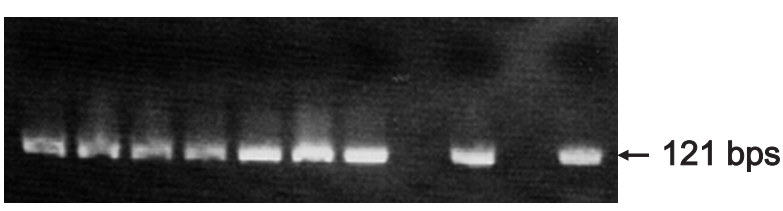

$\begin{array}{lllllllllll}1 & 2 & 3 & 4 & 5 & 6 & 7 & 8 & 9 & 10 & 11\end{array}$

Fig. 1. (A) Mean number of eggs per gram calculated by the Kato-Katz method. ${ }^{*}, P<0.01$. (B) Polymerase chain reaction results. Lanes $1-7,9$, and 11: positive samples; lanes 8 and 10: negative samples.

Table 1. Disease status and examination method

\begin{tabular}{clccc}
\hline Disease status & Examination method & No. of positive samples (\%) & No. of negative samples (\%) & Total No. \\
\hline \multirow{2}{*}{ acute } & Kato-Katz method & $15(30)$ & $35(70)$ & 50 \\
\multirow{3}{*}{ chronic } & PCR & $45(90)$ & $5(10)$ & 50 \\
& Kato-Katz method & $6(8.6)$ & $64(91.4)$ & 70 \\
& PCR & $68(97.1)$ & $2(2.9)$ & 70 \\
\hline
\end{tabular}


schistosomiasis group and 68 of 70 cases (97.1\%) in the chronic intestinal schistosomiasis group (Table 1). There was no significant difference in PCR sensitivity between the acute and chronic cases. The group 3 samples produced no PCR amplicons.

\section{DisCUSSION}

The development of single-dose oral drugs such as oxamniquine and praziquantel has helped to reduce schistosomiasis-related morbidity in easily diagnosable heavily infected patients using simple parasitological methods [20]. However, because the proportion of lowintensity infections has increased, more sensitive diagnostic methods are necessary [20]. PCR, an extremely sensitive and specific technique, is used widely to diagnose many infectious diseases including $S$. mansoni infections in humans [10]. The high sensitivity of the $S$. mansoni PCR assay is attributable to the 121-bp tandem repeats in its DNA sequence, which comprise at least $12 \%$ of its genome [15].

Our data shows that in the acute intestinal schistosomiasis group, the percentage of positive cases detected by the Kato-Katz method was $30 \%$, while the percentage detected by PCR was $90 \%$. In the chronic intestinal schistosomiasis group, the percentage of positive cases detected by the Kato-Katz method was $8.6 \%$, while that of PCR was $97.1 \%$. Thus, PCR is more sensitive at detecting both acute and chronic diseases than the Kato-Katz method. The PCR method diagnosed schistosomiasis more accurately in chronic disease cases where the Kato-Katz method failed to detect parasite eggs.

The Kato-Katz method is currently the recommended and most widely used technique for diagnosing $S$. mansoni infections. Its advantages are as follows: high specificity, simplicity of use, cost effectiveness, requirement of personnel with basic training only, and the ability to perform the test immediately in the field [11]. However, compared to PCR, this method is not sensitive enough to use in conditions of low parasite prevalence and low intensity of infection [10]. Although highly sensitive [10, 15, 16, 21, 22], PCR is very expensive, requires a laboratory set-up and trained personnel. Therefore, PCR cannot be expected to completely replace Kato-Katz methods at this stage.

Using PCR, detection of $1.3 \mathrm{fg}$ of $S$. mansoni genomic DNA, which is equivalent to less than the DNA found in a single cell of this multicellular parasite, was reported [20, 21]. Recently, the PCR sensitivity increased to 10 ag of DNA, which is equal in amount to less than one onethousandth of the DNA quantity contained in a parasite egg or cell, using conventional PCR [22]. A sensitive
PCR-enzyme-linked immunosorbent assay combination system for definitive diagnosis of schistosomiasis in feces has also been developed and evaluated [20]. Evaluation of the diagnostic sensitivity of this system showed that its ability to detect DNA in samples was consistent with the egg count in such samples, and the result obtained $(0.1534$ EPG) corresponded to a tiny part of a parasite egg [20].

The gold standard for Kato-Katz diagnosis is generally accepted to involve the analysis of three stools, with three slides per stool examined. Here, one fecal sample per subject was collected and analyzed by this method because the stool samples were collected on three successive days; this amounts to three fecal samples per subject, thus corresponding to the Kato-Katz method gold standard.

Taken together, our findings indicate that PCR is a far more sensitive diagnostic technique (especially for cases with low worm burdens such as chronic infection cases) for the detection of $S$. mansoni infection than the KatoKatz method, although the latter offers advantages over molecular and serological methods in terms of cost and ease of use.

\section{ACKNOWLEDGMENTS}

The authors would like to thank Assistant Professor Hend Abdelghany of the Department of Biochemistry, Faculty of Medicine, Minia University for her continuous advice on the technical aspects of this study.

\section{Conflict of Interest}

None to declare.

\section{REFERENCES}

1. Chitsulo L, Engels D, Montresor A, et al. The global status of schistosomiasis and its control. Acta Trop 2000; 77: 41-51.

2. Steinmann P, Keiser J, Bos R, et al. Schistosomiasis and water resources development: systematic review, metaanalysis, and estimates of people at risk. Lancet Infect Dis 2006; 6: 411-425.

3. Bruun B, Aagaard-Hansen J. The social context of schistosomiasis and its control: an introduction and annotated bibliography. World Health Organization (2008). Geneva, Switzerland. Available: http://whqlibdoc.who.int/ publications/2008/9789241597180_eng.pdf

4. Utzinger J, Raso G, Brooker S, et al. Schistosomiasis and neglected tropical diseases: towards integrated and sustainable control and a word of caution. Parasitology 2009; 136: 1859-1874.

5. Gryseels B, Polman K, Clerinx J, et al. Human schistosomiasis. Lancet 2006; 368: 1106-1118. 
6. Lammie PJ, Fenwick A, Utzinger J. A blueprint for success: integration of neglected tropical disease control programmes. Trends Parasitol 2006; 22: 313-321.

7. King $\mathrm{CH}$. Toward the elimination of schistosomiasis. $\mathrm{N}$ Engl J Med 2009; 360: 106-109.

8. Utzinger J, Zhou XN, Chen MG, et al. Conquering schistosomiasis in China: the long march. Acta Trop 2005; 96 : 69-96.

9. World Health Organization. Report on Schistosomiasis. Report of the Scientific Working Group meeting on Schistosomiasis. Geneva, 14-16 November, 2005. Available: http://whqlibdoc.who.int/hq/2006/TDR_SWG_07_eng.pdf

10. Pontes LA, Dias-Neto E, Rabello A. Detection by polymerase chain reaction of Schistosoma mansoni DNA in human serum and feces. Am J Trop Med Hyg 2002; 66: $157-162$.

11. Katz N, Chaves A, Pellegrino J. A simple device for quantitative stool thick-smear technique in Schistosomiasis mansoni. Rev Inst Med Trop Sao Paulo 1972; 14: $397-$ 400 .

12. Hassan MM, Badawi MA, Strand M. Circulating schistosomal antigen in diagnosis and assessment of cure in individuals infected with Schistosoma mansoni. Am J Trop Med Hyg 1992; 46: 737-744.

13. Lübeck PS, Wolffs P, On SL, et al. Toward an international standard for PCR-based detection of food-borne thermotolerant Campylobacters: assay development and analytical validation. Appl Environ Microbiol 2003; 69: 5664-5669.

14. Zarlenga DS, Higgins J. PCR as a diagnostic and quantitative technique in veterinary parasitology. Vet Parasitol 2001; 101: 215-230.
15. Hamburger J, Turetski T, Kapeller I, et al. Highly repeated short DNA sequences in the genome of Schistosoma mansoni recognized by a species-specific probe. Mol Biochem Parasitol 1991; 44: 73-80.

16. Hamburger J, Xu YX, Ramzy RM, et al. Development and laboratory evaluation of a polymerase chain reaction for monitoring Schistosoma mansoni infestation of water. Am J Trop Med Hyg 1998; 59: 468-473.

17. Moore KP, Wong F, Gines P, et al. The management of ascites in cirrhosis: report on the consensus conference of the International Ascites Club. Hepatology 2003; 38: 258-266.

18. Samuelson JC, Quinn JJ, Caulfield JP. Hatching, chemokinesis, and transformation of miracidia of Schistosoma mansoni. J Parasitol 1984; 70: 321-331.

19. Steiner JJ, Poklemba CJ, Fjellstrom RG, et al. A rapid one-tube genomic DNA extraction process for PCR and RAPD analyses. Nucleic Acids Res 1995; 23: 2569-2570.

20. Gomes LI, Dos Santos Marques LH, Enk MJ, et al. Development and evaluation of a sensitive PCR-ELISA system for detection of schistosoma infection in feces. PLoS Negl Trop Dis 2010; 4: e664.

21. Gomes AL, Melo FL, Werkhauser RP, et al. Development of a real time polymerase chain reaction for quantitation of Schistosoma mansoni DNA. Mem Inst Oswaldo Cruz 2006; 101 Suppl 1: 133-136.

22. Ferrer E, Pérez F, Bello I, et al. Polymerase chain reaction for the amplification of the 121-bp repetitive sequence of Schistosoma mansoni: a highly sensitive potential diagnostic tool for areas of low endemicity. J Helminthol 2014; 20: 1-5. 